Revue d'histoire de l'Amérique française

REVUE D.HISTOIRE DE L'AMÉRIQUE FRANÇAISE

\title{
Saint Antoine Daniel, martyr canadien (suite)
}

\section{Fernand Potvin}

Volume 9, numéro 2, septembre 1955

URI : https://id.erudit.org/iderudit/301708ar

DOI : https://doi.org/10.7202/301708ar

Aller au sommaire du numéro

Éditeur(s)

Institut d'histoire de l'Amérique française

ISSN

0035-2357 (imprimé)

1492-1383 (numérique)

Découvrir la revue

Citer cet article

Potvin, F. (1955). Saint Antoine Daniel, martyr canadien (suite). Revue d'histoire de l'Amérique française, 9(2), 236-249. https://doi.org/10.7202/301708ar d'utilisation que vous pouvez consulter en ligne.

https://apropos.erudit.org/fr/usagers/politique-dutilisation/ 


\title{
SAINT ANTOINE DANIEL, MARTYR CANADIEN
}

\author{
(Suite)* \\ Chapitre IV \\ FONDATION DU «SÉMINAIRE INDIEN 》 (1636)
}

Le projet du Père le Jeune - Le Père Daniel Revient de Huronie - DifficultéS AUX Trois-Rivières.

Ainsi, au moment où les missionnaires commençaient à élargir leur champ d'action et à utiliser des méthodes d'apostolat plus adaptées aux mœurs des indigènes, le Père Daniel abandonnait soudainement son œuvre pour revenir à Québec, accompagné de quelques jeunes sauvages. Sa présence en $\mathrm{Hu}$ ronie s'avérait pourtant de plus en plus nécessaire, car, depuis quelque temps, les Pères songeaient à établir une nouvelle résidence à l'intérieur du pays: «... ceste separation, écrit le P. François Le Mercier, nous fut vn peu sensible d'abord: car nous iugions dés lors, que pour trauailler plus efficacement à la conuersion de ces peuples, il nous falloit vne nouuelle habitation dans le cœur du pays, et le Père sembloit nous estre tout à faict necessaire pour cét effect, n'y ayant que luy qui pust, apres le R. Pere Iean de Brebeuf nostre Superieur, se desmesler aisément en la langue. Mais nous iugeasmes que de donner commencement à vn Seminaire de Ieunesse Huronne, estoit vne chose si auantageuse pour la gloire de Dieu, que nous auons passé par dessus ceste consideration, auec esperance que Dieu nous dénoüeroit bien tost la langue ... ». ${ }^{1}$

Cependant, ce n'était pas le seul sacrifice que les Pères devaient s'imposer. Cinq jours plus tard, «le 27. [juillet 1636] le P. Ambroise Dauost s'embarqua. Il sembloit necessaire en ces 74-92.

*Voir, notre Revue, vol. VIII (3): 395-414; (4) : 556-564; IX (1) :

1 F. Le Mercier, Q 1637, 103-4. 
commencemens, au cas que Dieu disposast du P. Daniel, que quelqu'vn se trouuast sur les lieux pour prendre sa place $»^{2}$ Mais quelle était donc l'importance de ce projet, puisque le Père Le Jeune, supérieur de la Mission canadienne, avait cru devoir dépouiller la Huronie des plus précieux auxiliaires du Père de Brébeuf ?

L'établissement d'un séminaire indien pour l'instruction et l'éducation des sauvages avait toujours hanté l'esprit inventif et entreprenant du Père Le Jeune. Ce projet n'était pas sans doute une création entièrement originale car déjà, les Récollets avaient songé à une entreprise analogue. ${ }^{3}$ De plus, le Père Le Jeune avait probablement entendu parler de la méthode utilisée par ses confrères Jésuites en Floride: ces derniers s'étaient empressés d'envoyer plusieurs Indiens dans un séminaire de la Havane afin de former peu à peu une jeunesse élite qui aiderait puissamment à la conversion de son milieu. ${ }^{4}$

Dès son arrivée au Canada en 1632, le Père Le Jeune, parlant des nations nomades qui habitent dans la région de Québec, déclare: "Le moyen à mon auis de les ayder, c'est de dresser des Seminaires, et prendre leurs enfans qui sont bien esueillez et fort gentils: on instruira le pere par le moyen des enfans $\gg .{ }^{5}$ Le Père Le Jeune prévoyait déjà qu'on pourrait très difficilement convertir les Algonquins à moins de les fixer dans des réductions ${ }^{6}$ ou de les instruire par l'intermédiaire des jeunes gens que l'on aurait arrêtés durant un ou deux hivers à Québec. L'année suivante, le Père Le Jeune perfectionne son plan en y ajoutant le projet d'un «seminaire de filles»; il s'était rendu compte que, pour faire œuvre durable, il faudrait que plus tard, mari et femme soient de même croyance, «car les garçons que

2 F. Le Mercier, Q 1637, 104g.

3 Rochemonteix, 1: 280.

4 Le fait est rapporté par L. Groulx dans « Missionnaires de l'Est en Nouvelle-France », Revue d'Histoire de l'Amérique Française, 3 (1949) : 58. 5 P. Le Jeune, Q 1632, 6d.

${ }^{6}$ C'est là une nécessité qui va s'imposer de plus en plus à son esprit. En 1634, il écrira: "Ie m'en rapporte, mais si ie puis tirer quelque conclusion des choses que ie vois, il me semble qu'on ne doit pas esperer grande chose des Sauuages, tant qu'ils seront errants: vous les instruisés auiourd'huy, demain la faim vous enleuera vos auditeurs, les contraignant d'aller chercher leur vie dans les fleuues et dans les bois », P. Le Jeune Q 1634, 11g. 
nous aurons éleuez en la cognoissance de Dieu venans à se marier à des filles ou femmes Sauuages accoustumées à courre dans les bois, leurs maris seront obligez de les suiure, et ainsi retomber dans la barbarie, ou bien de les quitter, qui seroit vn autre mal fort dangereux » $^{7}$

Vers ce temps-là, un incident lui fit prendre conscience d'une autre difficulté qui s'opposerait sérieusement à la réalisation de ses desseins. Un petit Français, s'amusant à jouer du tambour, avait brusquement frappé de son bâton un sauvage à la tête pour se délivrer de son importune curiosité. Aussitôt, grand émoi de part et d'autre: les Français veulent châtier l'enfant tandis que les sauvages réclament des présents pour effacer l'offense :

On fait venir le petit garçon; quand les Sauuages veirent que c'estoit tout de bon qu'on despoüilloit ce petit batteur de Sauuages et de tambour, et que les verges estoient toutes prestes, ils commencerent à prier qu'on luy pardonnast, alleguans que c'estoit vn enfant, qu'il n'auoit point d'esprit, qu'il ne sçauoit pas encor ce qu'il faisoit; mais comme on le vouloit chastier à toute force, l'vn d'eux se met tout nud, iette sa robe sur l'enfant, s'écriant à celuy qui le vouloit frapper; touche sur moy, si tu veux, mais tu ne le frapperas point: voila comme le pauure petit euada. Toutes les nations Sauuages de ces quartiers, et du Bresil, à ce qu'on nous témoigne, ne sçauroient chastier ni voir chastier vn enfant. ${ }^{8}$

Et le Père Le Jeune ajoute malicieusement: «... que cela nous donnera de peine dans le dessein que nous auons d'instruire la ieunesse $! \gg 9$

En 1634, dans un chapitre intitulé «Des moyens de conuertir les Sauuages », le Père Le Jeune développe un plan méthodique pour l'accroissement de la religion. Le premier de ces moyens est de maîtriser les Iroquois qui nuisent considérablement au prestige des Français; le second, d'aider les nomades à se fixer à demeure sur des terres cultivables; enfin, «le troisiesme

7 P. Le Jeune, Q 1633, $14 \mathrm{~d}$.

8 Ibid., 30g. .

9 P. Le Jeune, Q 1633, 30g. 
moyen d'estre bien-voulu de ces peuples, seroit de dresser icy vn seminaire de petits garçons, et auec le temps vn de filles, soubs la conduite de quelque braue maistresse, que le zele de la gloire de Dieu et l'affection au salut de ces peuples fera passer icy, auec quelques Compagnes animées de pareil courage ». ${ }^{10}$ Cependant, le séminaire ne sera d'abord établi que pour les jeunes Hurons que le Père de Brébeuf espère envoyer bientôt à Québec. ${ }^{11}$ Disposition très sage, car les Indiens étaient incapables de refuser quoi que ce fût à un enfant, à plus forte raison ne voudraient-ils pas le voir châtier en aucune manière. Comment, dans ces conditions, procéder à l'éducation des petits sauvages sinon en les tirant hors de leur pays, à l'abri de la tendresse excessive de leurs parents ? ${ }^{12}$ Au surplus, la présence de jeunes Hurons à Québec protégera celle des Pères et des Français en Huronie car les Indiens, par crainte de représailles, n'oseront pas leur faire subir de mauvais traitements. ${ }^{13}$

Mais l'exécution du projet se faisait attendre et les difficultés ne provenaient pas tant du mauvais vouloir des Hurons que de l'incapacité financière où se trouvait réduite la Mission. En 1635, un an après le départ du Père de Brébeuf et du Père Daniel pour la Huronie, l'infatigable supérieur de la Mission canadienne écrit encore: "La Residence de Nostre dame des Anges, appuyée principalement sur les liberalitez de Monsieur le Marquis de Gamache... a trois grands desseins pour la gloire de nostre Seigneur. Le premier, de dresser vn College pour instruire les enfans des familles qui vont tous les iours multipliant. Le second, d'établir vn Seminaire de petits Sauua-

$10 \mathrm{P}$. Le Jeune, Q $1634,12 \mathrm{~g}$; ce projet ne prendra forme qu'en 1639 alors que Marie de l'Incarnation viendra à Québec fonder le premier couvent d'Ursulines.

11 «Le Pere Brebeuf nous faict esperer que nous en pourrons auoir, s'il entre auec nos Peres dans ces pays bien peuplez, et si on trouue de quoy fonder ce seminaire », P. Le Jeune, Q 1634, 12d.

12 Dans une lettre à son Provincial, le Père Le Jeune lui fait part avec enthousiasme de son projet: " $\mathrm{Si}$ nous estions bastis, j'espererois que dans deux ans le P. Brebeuf nous envoiroit des enfants hurons; on les pourroit instruire icy avec toute liberté, estant esloignés de leurs parens. O le grand coup pour la gloire de Dieu si cela se faisoit $»$, P. Le Jeune au R.P. Provincial de France, 1634, Carayon, 153.

13 Voir P. Le Jeune, Q 1634, 12 d. 
ges, pour les éleuer en la foy Chrestienne. Le troisiéme, de secourir puissamment la Mission de nos Peres aux Hurons et austres Peuples sedentaires $\gg .{ }^{14}$

Comme tous les missionnaires de la Huronie, ${ }^{15}$ le Père Le Jeune croyait donc fermement pouvoir convertir cette nation en la renouvelant, pour ainsi dire, par sa jeune génération. $\mathrm{Ce}$ projet n'avait apparemment rien d'arbitraire ou de chimérique car, de tout temps, les idées et les traditions ont été enracinées moins profondément chez les enfants que chez les adultes. Soustraire un groupe de jeunes sauvages à l'influence de leur milieu paraissait donc le seul moyen praticable de leur faire acquérir de solides convictions religieuses. Une fois gagnés à la religion, ces jeunes Hurons deviendraient d'actifs foyers de vie chrétienne dans leur pays. Telle était la pensée du Père Le Jeune sur les moyens les plus efficaces d'établir l'Église parmi ce nouveau peuple.

Ayant reçu de son Provincial l'autorisation de «commencer un Séminaire, Dieu semblant disposer quelques bonnes âmes à le fonder $\gg,{ }^{16}$ le Père Le Jeune écrivit au Père de Brébeuf pour lui demander de choisir quelques jeunes sauvages, intelligents et sympathiques aux Français. Après avoir vaincu bien des résistances, on finit par en trouver douze. ${ }^{17}$ Pour les conduire à Québec et organiser ce collège nouveau genre, le Père de Brébeuf songea tout naturellement au Père Daniel, son meilleur compagnon d'apostolat, bien qu'il lui en coûtât de se séparer d'un collaborateur aussi précieux. Mais le choix s'imposait car, des cinq Pères qui se trouvaient alors en Huronie, il n'y avait que lui «Qui pust apres le R. P. Iean de Brébeuf nostre supérieur se demesler aisement en la langue $\gg, 18$ ou manier avec autant de tact le caractère capricieux des jeunes Hurons.

14 P. Le Jeune, Q 1635, 3d.

15 Voir ch. III, 48, 51-52.

16 P. Le Jeune, Q 1637, 55d.

17 Ibid., $55 \mathrm{~d}$.

18 F. Le Mercier, Q 1637, 103d; de son côté, le Père Le Jeune dira: «Le Pere Daniel venoit pour les dresser comme ayant déjà une assez bonne cognoissance de leur langue $\gg, P$. Le Jeune, Q 1636, $72 \mathrm{~g}$; voir aussi Latourelle, $1: 194$. 
Au moment où ces petits sauvages allaient s'embarquer dans les canots, l'affection bien compréhensible des mères pour leurs enfants menaça de faire échouer tout le projet: «... mais quand ce vint à partir, les meres et notamment les grandes meres, ne pouuoient laisser sortir leurs enfans, pour faire trois cens lieuës, et demeurer auec des Estrangers de façons de faire et de mœurs bien differentes des leurs ».19 Enfin, après avoir calmé bien des inquiétudes et rassuré de son mieux les parents éplorés, le Père Daniel dut se contenter de trois enfants au lieu des douze qu'on lui avait promis.

Mais son voyage devait être marqué par les difficultés bien plus grandes encore. Quelques jours avant d'arriver à l'«Isle», le Père Daniel avait rencontré les Pères Chastellain et Garnier qui montaient en Huronie. ${ }^{20}$ Par leur intermédiaire, il apprit que le «Général de la flotte » se préparait incessamment à s'embarquer pour la France. ${ }^{21}$ D'après son expérience, il prévoyait que les Hurons ne remettraient pas les trois jeunes sauvages entre les mains des Pères sans soulever auparavant d'interminables discussions. Aussi le Père Daniel, désireux de s'assurer l'influence considérable que du Plessis Bochard exerçait sur les Indiens, recommanda-t-il à ses gens de faire diligence.

Partis de la Huronie le 22 juillet, ${ }^{22}$ les canots hurons abordèrent à l'«Isle » des Algonquins au début du mois d'août. «Ces insulaires, comme l'explique le Père Le Jeune, voudroient bien que les Hurons ne vinssent point aux François, et que les François n'allassent point aux Hurons, afin d'emporter eux seuls tout le trafic; c'est pourquoy ils ont fait tout ce qu'ils ont peu pour nous boucher le chemin: mais comme ils craignent les François, ceux qui accompagnent les Hurons leur facilitent le chemin $\gg^{23}$ Cette année-là, le passage s'annonçait comme particulièrement difficile à cause de certaines pratiques superstitieuses faisant suite à la mort d'un chef de grande réputation surnommé

19 P. Le Jeune, Q 1636, 72g.

20 Les Pères Garnier et Chastellain étaient partis des Trois-Rivières le 21 juillet pour se rendre en Huronie. Voir ibid., 64g.

21 Ibid., $69 \mathrm{~d}$.

22 F. Le Mercier, Q 1637, 103g.

23 P. Le Jeune, Q 1636, 70; voir aussi Q 1633, 34g. 
《le Borgne », déjà connu pour son hostilité contre les Français. ${ }^{24}$ À son arrivée chez les Algonquins, le Père Daniel s'aperçut très tôt que ces derniers étaient loin d'être disposés à laisser passer cette nouvelle flottille de canots hurons. Craignant un retard peut-être considérable, il s'empressa d'expédier au «Général de la flotte » un messager auquel il remit en même temps une lettre assez circonstanciée expliquant au Père Le Jeune les motifs de son retard:

Ie demeure à l'Isle, en attendant le gros de la bande, tant des Hurons, que des Nipisiriniens. Les Sauuages de ce lieu auoient déjà renuoyé treize Canots de Hurons, leur defendant de passer aux François; mais leur Capitaine nommé Taratouan, ayant appris que ie descendois, a tenu ferme iusques à mon arriuée: car comme il est party deuant nous des Hurons, aussi sommes nous arriuez apres luy à l'Isle. Il m'a donc dit, que les habitans de cette Isle luy defendoient le passage; comme ie luy en demandois la raison, il m'a répondu, qu'on ne luy a dit autre chose, sinon que le corps d'vn Capitaine nouuellement mort, cestoit le Borgne de l'Isle, n'estoit pas encore caché; vous sçauez ce que c'est à dire, et partant que passer par deuant, c'estoit ietter du feu pour accroistre leur douleur, et irriter de nouueau les ieunes gens, qui sont fort fascheux et mutins. Ie luy ay reparty qu'il prist courage, que ie parlerois à ce Capitaine. En effet ie l'ay veu, il m'a fait assez bon accueil, Dieu mercy. Leur proposition estoit, qu'ils nous remeneroient nous autres François vers vous, mais que les Hurons eussent à s'en retourner. Or i'ay pris resolution de ne point passer, si les $\mathrm{Hu}-$ rons ne passent; ie le leur ay déjà promis, dont ils ont esté fort ioyeux. Ces difficultez leur font voir qu'il est important que nous demeurions dans leur Paiis, ce qu'ils cognoissent fort bien. I'ay prié le Capitaine de trouuer bon que i'enuoyasse deuant vn Canot pour donner aduis de nostre descente; c'est celuy qui vous porte ces lettres. Ie rencontray nos Peres le troisiéme d'Aoust, trois iournées au dessus de l'Isle, ils estoient tous deux chaussez dans leurs

24 Voir ch. II, 28. 
Canots sans ramer, ce qui me fait penser qu'ils sont doucement traittez; cela me fit faire pour leur gens, ce que ie n'auois pas encor voulu faire pour les miens, ce fut de leur faire present d'vne herbe qu'ils adorent, et que nous n'aymons point; c'est du Petun, dont la cherté est grande cette année. Ie voudrois bien en estre quitte pour dix fois autant à l'Isle, et vous voir au plustost auec de ieunes Hurons; ie n'épargneray rien pour ce sujet. Cette affaire est trop importante. De douze petits enfans, qui m'auoient promis de me suivre auec le consentement de leurs parens, ie n'en ay que trois auec moy, dont l'vn est petit fils d'vn fort grand Capitaine; i'en espere assez de grandelets, si vous en voulez, nous les verrons ensemble, quand i'auray le bonheur de vous voir; les petits ont de la peine à quitter leurs meres pour faire trois cens lieuës. I'écry à Monsieur du Plessis qu'il y a peu de Canots, mais qu'ils portent tres grande quantité de marchandises. Ie vous recommande les porteurs, que mes promesses s'il y a moyen se trouuent veritables; cela est de consequence.

De l'Isle ce septiéme d'Aoust, à la lueur d'vne écorce brûlante; ce sont les chandelles et les flambeaux du Païs. ${ }^{25}$

Toute prosaïque qu'elle paraisse, cette lettre du Père Daniel nous révèle quand même plus d'un aspect de sa personnalité. On y remarque d'abord une énergie qui en imposait même aux sauvages: treize canots de Hurons ont déjà rebroussé chemin, mais leur capitaine, à la nouvelle que le Père Daniel arriverait bientôt, change d'avis et décide de profiter de la puissante protection de la Robe Noire. Le Père Daniel, dès son arrivée, s'empresse de le rassurer et va lui-même discuter avec le capitaine de l'« Isle ». Malgré les propositions peu encourageantes que ce dernier lui fait, le Père Daniel ne se décourage pas : «Or i'ay pris resolution de ne point passer, si les Hurons ne passent, ie le leur ay déjà promis, dont ils ont esté fort ioyeux ». Mais voici qu'il s'avise de dépêcher aux Trois-Rivières un canot pour avertir le «Général de la flotte » et le Père Le Jeune de son retard imprévu. Plein

25 Lettre du Père Antoine Daniel au Père Paul Le Jeune, «Isle 》 des Algonquins, le 7 août 1636, cité par le P. Le Jeune, Q 1636, 69-70. 
d'assurance, il informe les Algonquins de son dessein: "I'ay prié le Capitaine de trouuer bon que i'enuoyasse deuant vn Canot pour donner aduis de nostre descente ».

Âme énergique mais âme charitable aussi. Trois jours avant d'arriver à l'« Isle », il a rencontré les Pères Chastellain et Garnier qui montaient en Huronie. À les voir confortablement assis dans le canot, sans aviron, leurs chaussures aux pieds, ${ }^{26}$ le Père Daniel devine que les sauvages qui les conduisent sont bien disposés à leur égard; afin de les garder aussi bienveillants envers leurs passagers, il leur fait don d'une réserve de «petun». Le même présent, fait à son équipage, aurait pu le dispenser de manier l'aviron, mais il préférait adoucir le voyage des autres plutôt que se procurer à lui-même quelque soulagement.

De nouvelles, il ne donne que celles-là qui intéressent de près la tâche qu'on lui a confiée: le nombre de séminaristes, sa détermination à les amener jusqu'aux Trois-Rivières malgré tous les obstacles. À peine quelques mots: «quand i'auray le bonheur de vous voir » nous rappellent qu'un homme se tient derrière ces lignes, un homme qui, depuis deux ans, vit en pleine sauvagerie et va bientôt retrouver un milieu civilisé, sympathique. S’il parle peu de ce bonheur bien légitime, c'est qu'il le considère trop personnel, presque égoïste; le cœur d'apôtre qui vit en lui l'invite sans cesse à ne plus penser à lui-même.

Mais bientôt allait se présenter une occasion qui ferait encore appel à son inépuisable charité. Délivrés des ennuis que les Algonquins de l'«Isle» leur avaient causées, le Père Daniel et ses Hurons descendaient sur les eaux rapides de l'Outaouais en direction des Trois-Rivières lorsque, arrivés à la Petite-Nation des Algonquins, on leur apprit qu'un prisonnier Iroquois était sur le point d'être supplicié. Le Père Daniel ne perd pas un instant: il fait quelques présents aux gardes du condamné afin de pouvoir s'approcher de lui plus librement. En quelques mots, il lui expose sa triste condition, les peines infiniment plus douloureuses encore qui l'attendent peut-être au sortir de la vie, il lui fait regretter ses fautes passées et un instant plus tard, les

26 Voir les explications que le Père Le Jeune donne à ce sujet dans $\mathrm{Q}$ 1636, 70-71; voir aussi L. Campeau, « Voyageurs et martyrs », Lettres $d u$ Bas-Canada, 2 (1948): 11-23. 
eaux du Baptême regénéraient l'âme de ce malheureux Indien avant qu'on eût commencé à le torturer. ${ }^{27}$

Quelques jours plus tard, le Père Daniel et une partie des Hurons arrivaient en vue des Trois-Rivières. Dès qu'ils eurent aperçu les canots indiens sur le fleuve, le Père Le Jeune, le «Général de la flotte » et les quelques Français de l'endroit s'empressèrent de descendre du fort pour aller les accueillir. C'était le 19 août $1636 . .^{28}$ Il y avait un peu plus de deux ans que le Père Le Jeune avait vu les premiers missionnaires s'embarquer pour la Huronie. Le Père Daniel était maintenant le premier qu'il voyait revenir des «pays d'en Haut». Le miracle s'était donc réalisé: désormais, malgré tous les obstacles, les fatigues, les dangers d'un si long voyage, des communications régulières pourraient assurer le progrès constant de la Mission. Toutes ces pensées, jointes à la perspective du séminaire indien qui allait bientôt s'organiser, se présentaient spontanément à l'esprit du Père Le Jeune et c'est d'un ton légèrement ému qu'il raconte l'arrivée du Père Daniel:

A la veuë du Pere Daniel, nostre cœur s'attendrit: il auoit la face toute gaye et ioyeuse, mais toute défaite; il estoit pieds nuds, l'auiron à la main, couuert d'vne méchante soutane, son Breuiaire pendu au col, sa chemise pourrie sur son dos. Il salüa nos Capitaines et nos François; nous l'embrassasmes, et l'ayans conduit en nostre petite chambre, apres auoir beny et adoré nostre Seigneur, il nous raconta en quel point estoient les affaires du Christianisme aux Hurons, me rendant les Lettres et la Relation qu'on enuoyoit de ce Païs, et nous obligeant à chanter vn Te Deum, en action de graces des benedictions que Dieu va versant sur cette Nouuelle Eglise. Ie ne parleray point des difficultez de son voyage, tout cela est déjà dit; ce luy estoit assez d'auoir baptisé vn pauure miserable qu'on menoit à la mort, pour adoucir tous ses trauaux. ${ }^{29}$

«La face toute gaye et ioyeuse, mais toute defaite »; ainsi, après deux ans seulement passés chez les Hurons, le Père Daniel

27 P. Le Jeune, Q 1636, 27 ; F. Le Mercier, Q 1637, 119g.

28 P. Le Jeune, Q 1636, 71g.

29 Ibid., 71. 
porte sur son visage les traits qu'y ont creusés la fatigue des longs voyages, l'étude assidue de la langue et, par-dessus tout, sa douloureuse impuissance à convertir les Hurons. Malgré cet échec, il a conservé un visage radieux qu'illumine encore la joie du retour. Son premier geste est d'aller rendre hommage à son Maître et Seigneur; c'est seulement après cet acte d'adoration qu'il commence à raconter « en quel point estoient les affaires du Christianisme aux Hurons ». Habitué à ne pas faire grand cas de lui-même, ses premières paroles ne disent rien de sa personne dont l'aspect misérable devait pourtant exciter une admiration respectueuse. Détail qui resta sans doute inaperçu mais qui révèle, à sa manière, un aspect de son âme. Un dernier trait enfin nous fait retrouver cette énergie presque autoritaire, que nous avions rencontré plus haut: «... il nous raconta... nous obligeant à chanter un Te Deum en action de grâces des bénédictions que Dieu va versant sur cette nouvelle Église». Sa gratitude pour les bienfaits reçus, il désire que tous en rendent avec lui témoignage à Dieu, reconnaissant par là que tous ont participé à son œuvre.

Aux curieux qui lui demandaient des nouvelles de son voyage, comme cela se pratique si naturellement, le Père Daniel n'a pas dû faire de longues réponses car le Père Le Jeune résume toutes ses explications dans cette courte phrase: «...ce luy estoit assez d'auoir baptisé vn pauure miserable qu'on menoit à la mort, pour adoucir tous ses trauaux ». Autre indice qui dévoile sans éclat l'humilité du Père Daniel et surtout la charité vivante qui l'habitait tout entier.

On se souvient que, dans sa lettre écrite de l'«Isle», le Père Daniel avait prévenu son supérieur de l'arrivée de trois séminaristes. ${ }^{30}$ C'était déjà beaucoup pour les ressources dont la Mission pouvait disposer mais comme il était difficile de commencer avec un nombre trop réduit, le Père Le Jeune désirait malgré tout en réunir une demi-douzaine, se confiant en la Providence pour subvenir à leurs besoins. ${ }^{31}$ Le Père de Brébeuf et

30 Voir ch. IV, 61-62.

31 P. Le Jeune, Q 1636, 71g. 
le Père Daniel avaient déjà éprouvé au départ de la Huronie jusqu'à quel point la tendresse des mères pouvait faire obstacle à leur projet: de douze séminaristes qu'on leur avait promis, trois seulement reçurent de leurs parents l'autorisation de s'embarquer. ${ }^{32}$ Aux Trois-Rivières, ce fut au tour des pères à s'opposer: «... mais quand ils furent arriuez, les peres de ces enfans reculoient, et cherchoient mille excuses. Le pauure Pere Daniel alloit et venoit de tous costez, amadoüoit les vns, faisoit quelques presens aux autres, et apres tout cela, il se vit quasi maistre sans écoliers, et pasteur sans oüailles $\gg^{33}$ Des trois séminaristes qui étaient descendus avec lui, il n'en restait plus qu'un seul, nommé Satouta, lequel avait promis de persévérer "dans la resolution qu'il auoit prise de se faire instruire et de demeurer auec nous $\gg .{ }^{34}$

La situation était vraiment critique. En effet, si l'on ne réussissait pas à lancer le séminaire indien cette année-là, le projet si longtemps médité par le Père Le Jeune risquerait fort d'être abandonné définitivement car les Hurons céderaient difficilement plus tard sur un point où ils auraient déjà triomphé. Une intervention vigoureuse s'imposait.

Le Père Le Jeune se concerta avec le Père Daniel et du Plessis Bochard, afin d'organiser une 'dernière assemblée à laquelle on invita tous les Hurons descendus pour la traite. Après leur avoir offert de nombreux présents en témoignage de l'amitié des Français, du Plessis Bochard se leva et fit demander aux Hurons

s'ils nous aymoient autant que nous les aymions; ils répondirent, qu'en effet ils nous aymoient. D'où vient donc que vous ne témoignez pas vostre amitié ? Vous donnez des robes de Castors aux François, et ils vous donnent des haches et d'autres marchandises: tout cela s'appelle trafiquer; ce ne sont point les marques du vray amour que ie recherche. Mais s'entre-visiter, s'entre-secourir, aller les vns dans le païs des autres, s'allier par ensemble comme les doigts de la main: ce sont des actes d'amitié; c'est

32 P. Le Jeune, Q 1637, 55d.

33 P. Le Jeune, Q 1636, 72g.

34 P. Le Jeune, Q 1637, 55d. 
ce que nous faisons, nous allons dans vostre païs, nous y enuoyons nos Peres, nos Maistres, ce que nous auons de plus cher, ceux qui nous enseignent le chemin du Ciel, et pas vn de vous ne veut demeurer auec nos François. Pourquoy ne vous confiez vous pas autant en nous, comme nous nous confions en vous ? ${ }^{35}$

Le Père Le Jeune avait fait asseoir Satouta entre lui et le Père Daniel; du Plessis Bochard témoigna alors publiquement la considération qu'il portait au jeune Huron en offrant à sa bourgade un nouveau présent. Bien plus, il remit à Satouta l'honneur de présider le grand festin d'adieu; quant à lui, il devait partir immédiatement pour la France. Bref, le «Général de la flotte 》 produisit, avec son habileté coutumière, ${ }^{36}$ les arguments les plus capables d'ébranler l'obstination des sauvages.

Ces derniers ne savaient plus quoi répondre ni comment résister à tant de promesses. Pourtant l'assemblée se dispersa sans qu'aucune décision favorable fût venue mettre fin aux débats. Les Pères commençaient déjà à perdre l'espoir de fonder le Séminaire cette année-là lorsqu'un capitaine, se ravisant, entreprit de haranguer les siens, leur disant: «qu'il deuoient auoir honte de se monstrer moins affectionnez aux François, que la Nation des Ours, d'où estoit Satouta; que nous estions bons et courtois, qu'il n'y auoit point de danger de demeurer avec nous $\gg .{ }^{37} \mathrm{Et}$ sur l'heure, il intime l'ordre à deux jeunes gens de rester avec les Français, ce que les intéressés, ravis de cette nouvelle aventure, acceptèrent avec joie. On en vint aussitôt donner avis au Père Daniel qui s'empressa de communiquer l'heureuse décision au Père Le Jeune et à du Plessis Bochard. ${ }^{38}$

Le lendemain matin, quelques Hurons arrivèrent au fort afin de présenter au Père Daniel les deux nouveaux séminaristes. Le père de l'un d'eux lui fit ainsi ses recommandations: "Mon fils, sois constant, ne desiste point de ta resolution; tu t'en vas auec de bonnes personnes, tu ne manqueras de rien auec ces gens-là ; ne prends rien sans le congé d'Antoine; c'est ainsi qu'ils

$35 \mathrm{P}$. Le Jeune, Q 1636, $72 \mathrm{~d}$.

36 Voir par exemple le rôle décisif qu'il joua lors du premier départ pour la Huronie, ch. III, 33 ; cf. Q $1635,24 \mathrm{~g}$.

37 P. Le Jeune, Q 1636, 73d.

38 Ibid., 73 d. 
appellent le Père Antoine Daniel; ne frequente point les Montagnés, mais les François seulement; sur tout obey à ceux qui portent des habits noirs, auec lesquels tu dois demeurer $\gg^{39}$ Le Père Daniel reçut les deux jeunes Hurons avec toute la cordialité possible et distribua encore quelques présents à leur famille. À quelques jours de là, le "Général de la flotte », incommodé par son état de santé, mit la dernière main à ses préparatifs de départ. ${ }^{40}$ Pour manifester une fois de plus aux sauvages quelle considération les autorités françaises avaient à l'égard des protégés des Pères, du Plessis Bochard fit monter le Père Daniel et ses élèves sur son propre navire. ${ }^{41}$ Lorsqu'ils mirent à la voile, les parents des trois jeunes séminaristes leur adressaient encore d'éloquentes recommandations : « Il faisoit beau voir leurs parens les apostrophans et leur recommandans d'auoir courage, de ne rien prendre parmy nous; que ce n'estoit point nostre coustume d'estre larrons; bref, ils firent cette action auec tant de témoignage d'amour, que tous nos François en estoient consolez $\gg .^{42}$

Trois séminaristes: Malgré ce maigre résultat, le projet du Père Le Jeune entrait enfin dans sa phase de réalisation et cette part, non la moindre, était confiée au Père Daniel. Ces trois jeunes Hurons, il devait les discipliner, les transformer, leur faire adopter, au moins partiellement, les mœurs européennes, les habituer à vivre «à la françoise », car c'était ainsi qu'à ce moment, on concevait leur éducation. ${ }^{43}$ Par-dessus tout, il devait leur apprendre à devenir d'excellents chrétiens, mieux encore, il devait leur former un cœur d'apôtre afin qu'à leur retour en Huronie, ils fussent de vrais témoins de la religion. En vérité, le Père Daniel entreprenait là une tâche assurément très noble, mais dont la réalisation paraissait bien ardue.

Fernand Potvin, s.j.

\section{(à suivre)}

39 Ibid., 73d.

40 Ibid., $74 \mathrm{~g}$.

$41 \mathrm{P}$. Le Jeune, Q 1636, 74g; voici comment le Père Le Jeune apprécia la conduite de du Plessis Bochard dans cette affaire: « Monsieur le General de la flotte s'en retourne auec cette loüange deuant Dieu, de n'auoir rien oublié icy pour la gloire de sa Majesté », ibid., $74 \mathrm{~g}$.

42 Ibid., $74 \mathrm{~g}$.

43 P. Le Jeune, Q 1637, 56d. 